\title{
Konviviale Integration in postmigrantischen Gesellschaften
}

\author{
Naika Foroutan
}

»Nicht Verhältnisse von Vertrautheit, sondern der Umgang unter Fremden wird zum Ausgangspunkt der Überlegungen gemacht«, schreiben Frank Adloff und Volker Heins in ihrer Einleitung zum vorliegenden Band über das konvivialistische Manifest und leiten über zu der Frage: »Wie zusammenleben, ohne sich gegenseitig zu schaden?« Reicht dieses Minimalziel - sich keinen Schaden zuzufügen - aus, um stark heterogene, von Debatten um Migration geprägte Gesellschaften vor der herausfordernden Polarisierung durch rechtspopulistische Parteien zu bewahren, die derzeit in 18 europäischen Nationalparlamenten vertreten sind? In postmigrantischen Gesellschaften, die politisch anerkannt haben, dass sie Einwanderungsländer geworden sind und Migration ein konstituierendes Element der Gesellschaft bildet, beanspruchen Migranten und ihre Nachkommen Teilhaberechte auf der Basis des Gleichheitsgrundsatzes der Demokratie. Dies führt zu Aushandlungen um Rechte, Positionen und Privilegien. Ambivalenzen rund um Identitätspolitiken und der Sehnsucht nach Normalisierung prägen die politischen Debatten ebenso wie der Antagonismus zwischen Migrationsbefürwortern und Migrationsgegnern. Neue Narrative entstehen, während die alten noch verteidigt werden und noch ältere erneuert gegen beide antreten. Die postmigrantische Gesellschaft ist ambivalent und unübersichtlich mit dem Versprechen einer radikalen über das >Migrantischeく hinausweisenden Utopie der Gleichheit, die jenseits der Herkunft verhandelt wird, weil Herkunft in der zunehmenden Unübersichtlichkeit und Heterogenität der Generationen verschwimmt. Die Rhetorik der Rechtspopulisten bietet eine Reduktion dieser Unübersichtlichkeit an, eine Rückkehr zur Homogenität oder einen sortierten Ethnopluralismus und zu Werten, die >Europa<, den $>$ Islam< und Einwanderung als Bedrohung der kollektiven Identität verwerfen. Daraus entsteht ein Kampf gegen Gesellschaftsformen, die von Vielfalt durch Migration geprägt sind, und deren Unübersichtlichkeit eine erhöhte Ambiguitätstoleranz voraussetzt. 
Im konvivialistischen Manifest, das beansprucht, »die Umrisse einer anderen möglichen Welt zu skizzieren«(S. 36), taucht das Wort Migration nur ein einziges Mal auf. Dies geschieht in der Einleitung, in der auf gegenwärtige Bedrohungen wie »die Klimaerwärmung, die Katastrophen und die gigantischen Migrationsströme, die sie nach sich ziehen werden«, verwiesen wird (S. 40). Migration als Kernkomponente von Mobilität, von dynamischer Umgestaltung der Einwanderungsgesellschaften, Normalitätsphänomen und dominantem Konfliktdiskurs findet keinen Eingang in die Überlegungen der vierzig Intellektuellen, die als Erstunterzeichner des Manifests auftreten. Stattdessen wird viel und mit maximaler Unverbindlichkeit vom Verhältnis von Menschen und Gruppen untereinander gesprochen. »Konvivialität braucht die Autonomie der Gesellschaft, die sich durch zivilgesellschaftliche Assoziationen realisiert«, (S. 36) fasst Frank Adloff zusammen.

Aber reicht es aus, auf zivilgesellschaftliche Assoziationen zu setzen, wenn die Polarisierung bereits institutionell und politisch strukturiert ist, wie es nicht zuletzt in der Parteienstruktur, sondern auch anhand zivilgesellschaftlich aufgebauter Institutionen, wie Bürgerbüros, Online-Portalen, Radiosendern rechtspopulistischer Parteien in Europa zu beobachten ist? Rechtspopulistische Positionen und Stimmen sind also keineswegs mehr assoziativ zivilgesellschaftlich organisiert, was der 25 Prozent-Erfolg des Front National auf dem Weg ins Europaparlament nahelegt, ebenso wie die 27 Prozent der UKIP in Großbritannien oder die 26,6 Prozent für die Dänische Volkspartei. Der deutliche Anstieg dieser nationalkonservativen Parteien und hier sei weniger auf die seit Jahren um 20-25 Prozent etablierten wie die SVP in der Schweiz oder die FPÖ in Österreich verwiesen, sondern eher auf die Schwedendemokraten, Wahren Finnen, Vlaams Belang, die 52 Prozent für FIDESZ in Ungarn und die PiS in Polen, die nun den Präsidenten stellt, zeigt: Die Rechtspopulisten besitzen sehr wohl eine institutionelle Macht und nicht nur eine assoziative und geben eine Agenda vor, die auf ihre Art auch auf ein gelingendes $\mathrm{Zu}$ sammenleben ausgerichtet ist - allerdings auf Kosten von Minderheiten und mit dem Ziel der massiven Einschränkung von Einwanderung, globaler und europäischer Kooperation und Wertevielfalt. Die zunehmend offensiv formulierten Antworten der Migrationsgegner lassen ein bloßes Abwarten, wohin die Gesellschaft sich entwickelt, fragwürdig erscheinen. Die zunehmenden Positionsgewinne rechtspopulistischer Parteien führen dazu, sich die Frage »Wie wollen wir zusammenleben? « auch unter dem Aspekt der zunehmenden Migrationsbewegungen deutlich zu stellen - und eine konstruktive Antwort zu formulieren, wenn man sich den einfachen Antworten der Rechtspopulisten entgegenstellen will. Hier gibt es im konvivialistischen Manifest eine Leerstelle. 


\section{SiNNSUCHE}

Postmigrantische Gesellschaften sind von Aushandlungen, Ambivalenzen, Antagonismen und Allianzen gekennzeichnet. Sie sind keine utopischen Gemeinschaften, in denen Rassismus und Ungleichheit überwunden sind. Vielmehr sind sie geprägt von Konflikten zwischen jenen, die unter Demokratie gleiche Rechte für alle Bürger verstehen und jenen, die Vorrechte nur für die jeweils eigene Gruppe beanspruchen. Wir beobachten hier zunehmend eine Spannung zwischen kognitiver und affektiver Wahrnehmung von Zusammenleben: Während der Sachverständigenrat deutscher Stiftungen für Integration und Migration (SVR) in Deutschland von einem Integrationsoptimismus ausgeht (SVR 2014) und weitere Studien für die deutsche Bevölkerung eine Offenheit gegenüber Einwanderung und Diversität konstatieren (GMF 2013), sind Abwehrhaltungen und Stereotype gegenüber sichtbaren Minderheiten auf einem hohen Niveau stabil (Decker/Kiess/Brähler 2014; Foroutan et al. 2015). Auch gegenüber >nicht sichtbaren Minderheiten wie (säkularen) Juden oder Homosexuellen hält sich eine latente emotionale Abwehr. Man könnte geneigt sein zu denken: Solange sie sich »keinen Schaden zufügen«, sollen die Menschen doch denken, was sie wollen - jeder hat ein Recht auf Antipathie, wir sind nicht verpflichtet, einander zu lieben! Gleichzeitig müssen wir uns fragen, ob demokratische Grundwerte wie Gleichheit und Gleichwertigkeit gänzlich unabhängig von Empathie und übergeordneter Sinngebung tragfähig bleiben oder aber implodieren, wenn die Gleichgültigkeit des Nebeneinanderlebens von dem berauschenden Angebot der Rechtspopulisten überrumpelt wird?

In den 1970er Jahren formulierten vor allem französische Philosophen wie Jean-François Lyotard ihre Kritik an übergeordneten, sinnstiftenden Erzählungen, von denen angenommen wurde, dass sie immer nur exklusiv seien und konkurrierende Wahrnehmungs- und Wahrheitsansprüche grundsätzlich ausschlössen. Dass sie auch miteinander existieren und komplementär sowie kompetitiv zu einander funktionieren können, wurde weniger mitgedacht. Allerdings ist die parallele und gleichzeitige Präsenz von multiplen Großerzählungen (Religion, Säkularismus, Neoliberalismus, Kommunismus etc.) in heterogenen Gesellschaften augenscheinlich, wo unterschiedliche religiöse, werteorientierte, ökonomische und politische Konzepte teilweise miteinander konkurrieren, aber auch überlappend und verschränkt neben- und miteinander bestehen können. Gleichzeitig gibt es dominante Großerzählungen, die stark homogenisierend und ausschließend sind (z.B. Islamismus oder Nationalismus) und Zuspruch bis in die Mitte der Gesellschaft hinein erhalten man denke z.B. an die Ergebnisse der Studien der Friedrich-Ebert Stiftung, die deutlich fremdenfeindliche, islamophobe und homogenisierende Zusprüche in der Mitte der Gesellschaft aufzeigten (Decker/Kiess/Brähler 2014). Auch Hinwendungen junger Menschen zu radikalen und extremistischen islamis- 
tischen Gruppierungen zeigen die Anziehungskraft exklusivistischer Großerzählungen, die strukturierend und gesellschaftserklärend auf Menschen wirken. Daher stellen die Autoren des konvivialistischen Manifests zurecht die Frage, wie man »mit den Gefahren moralischer und politischer Art, die man als anthropisch bezeichnen könnte (S. 45) umzugehen habe?

Aber wenn die Autoren sich für »ein Maximum an Pluralität, das noch möglich ist, ohne den Zusammenhalt zu gefährden « (S. 27) aussprechen, wie Adloff zusammenfasst, wird deutlich, dass sie sich nicht trauen, zu beschreiben, was sie mit der mitschwingenden Einschränkung konkret meinen? Denn durch den ganzen Text hindurch ist die Unentschiedenheit zu spüren, dass die Autoren sich auf der einen Seite nach einem sinngebenden Metanarrativ sehnen, es aber auch gleichzeitig ablehnen, ein solches zu formulieren. So beschreiben sie einen diaphanen Möglichkeitsraum, in dem »ein gleiches Recht auf Verwurzelung wie auf Entwurzelung, auf das Gleichheitsrecht der Kulturen und zugleich auf ihr Recht, sich voneinander radikal zu unterscheiden«, (S. 27) skizziert wird. Die Frage, vor der sich die Autoren und mit ihnen wir Intellektuellen, die in der Dekonstruktion und der Hybridität, der Dritten Räume und der situativen Identitäten sozialisiert sind, drücken, lautet, ob heterogene Gesellschaften nicht doch einer übergeordneten politik- und handlungsleitenden Großerzählung bedürfen, die sie in ihrem politischen und narrativen Selbstbild strukturiert, weil die Heterogenität als pures Nebeneinander nicht als sinngebend empfunden wird, wenn ihr kein sinnstiftender Endpunkt vorausgeht, auf den diese Vielheit zuläuft und der die Entwicklung der Gesellschaft auf diesem Weg begründet. Und vor allem weil diese Pluralität, die wir als Intellektuelle ersehnen, weil wir es als bereichernd, herausfordernd und anregend empfinden, uns in der Vielheit immer wieder zu verlieren und neu zu definieren, viele Menschen überfordert und verunsichert, die nun ein Angebot für Eindeutigkeiten und zur Ambiguitätsreduktion von rechten Populisten und Islamisten bekommen, während wir noch weiter mit Dekonstruktionen beschäftigt sind. Die Angebotsunterbreiter wie Marine Le Pen, Geert Wilders, die Schwedendemokraten, die UKIP, die AfD oder Pegida warnen vor Parallelstrukturen, Chaos, Unordnung und Bezugslosigkeit, dort wo wir Mehrfachzugehörigkeiten, Fluidität und Code-Switching hochhalten. Die Salafisten und Islamisten winken mit wahren Identitäten, während wir die Unentschiedenheit und die Hybridität als Normalität betrachten.

Die große Frage, die sich nun an uns stellt - und zu der die Autoren des konvivialistischen Manifests sich nicht vorwagen - ist, ob es auch Großerzählungen geben kann, die sinnstiftend und strukturierend auf Politik und Zivilgesellschaft Einfluss nehmen können, ohne exklusiv und homogenisierend zu wirken. Dabei stellt sich wieder einmal die Frage, ob ein Verfassungspatriotismus als Basis ausreicht, um heterogene Gesellschaften - etwa die deutsche Gesellschaft - zukünftig politisch weiterzudenken. Braucht es vielleicht zu- 
sätzlich einen sinnstiftenden Endpunkt, ein handlungsleitendes Motiv oder Leitbild, welches politisch definiert, wie dieses neue heterogene Deutschland sich erzählt? Wer erzählt und entwirft dieses Motiv? Und lässt ein solches Leitbild - eine Neudefinition des pluralen postmigrantischen Deutschlands - nicht auch wieder >Andere< zurück, wie Pegida oder Salafisten? Wenn Soziologen sagen, dass es keine kollektive soziale Identität geben kann ohne die Existenz einer Outgroup, dann müssen wir entweder Heterogenität mit dem Fortbestand von Outgroups zusammendenken. Oder wir denken weniger sozialpsychologisch und orientieren uns am philosophischen Ansatz Martin Bubers und seinem »Ich « in Gegenüberstellung zum »Du«. So könnte ein neues deutsches »Wir«, zwar immer noch nur in Kombination mit einem »Ihr« gedacht werden, aber dieses »Ihr« wäre keine dichotome Ingroup-Outgroup-Kategorie, sondern Bezugspunkt eines sinnstiftenden Narratives in einer dynamischen Integrationslogik, die danach strebt, Ungleichheit in der Gesellschaft abzubauen und Gleichwertigkeit als Basis eines Zusammenlebens in einer Gesellschaft zu sehen, die von Vielheit, Heterogenität und Pluralität gekennzeichnet ist. Die Integration in dieses Leitmotiv bzw. Metanarrativ der Vielheit oder der pluralen Demokratie wäre dann eine Zielperspektive, die für die Politik handlungsleitend und für die Zivilgesellschaft sinnstiftend wirken könnte.

\section{Integration als Weg - Konvivialismus als Ziel?}

Das Problem, Integration als politisches Leitmotiv für eine Form des Zusammenlebens anzubieten, die auf Vielheit und Gleichwertigkeit gründet, verweist auf den Begriff der Integration selbst. Dieser hat sich in den letzten 35 Jahren - seit der Veröffentlichung des sogenannten Kühn-Memorandums (Kühn 1979) - in Deutschland in einem steten Wechselspiel zwischen Wissenschaft, Politik, Zivilgesellschaft und Medien als Vorstellung einer Kerngesellschaft etabliert, an welche sich Einwanderer oder Migranten anzupassen haben. Problematisch an dieser Perspektive sind zum einen der unilaterale Bezugspunkt sowie die suggerierte Bringschuld der Migranten, was den Blick von gesellschaftlichen Aufgaben weglenkt. Der Begriff wird dadurch seit Jahrzehnten einseitig verwendet. Auf diese Weise ist der Integrationsbegriff paradoxerweise selbst zu einem Integrationshemmnis und zu einem Exklusionsbegriff geworden. Das Paradoxon der Integrationsfrage besteht heute darin, dass mit dem Anstieg struktureller und sozialer Integration und mit einer zunehmenden Etablierung von Migranten und ihren Nachkommen in der bürgerlichen Mitte sowie in Elitepositionen, die etablierten Eliten weitere Hürden der Integration einbauen und Schließungen vornehmen. Sie erweisen sich also selbst als desintegrativ, was mit einer Angst vor Verteilungskämpfen zusammenhängen kann: »Die Migranten, die zu den besten Aspiranten auf Integration zählen, 
sind bevorzugt Ziel von Stigmatisierung, bedrohen sie doch vermeintlich am stärksten den Status der Einheimischen, « so der Frankfurter Soziologe Ferdinand Sutterlüty (2010). Die Distinktionsmechanismen, die Ungleichheiten trotz struktureller und sozialer Aufstiege symbolisch zementieren, wirken vor allem mit dem Argument der »kulturellen Inkompatibilität« von Migranten weiter, weswegen eine stärkere kulturelle Identität eingefordert wird, die aber gleichzeitig negiert wird. Hierbei konzentriert sich die Debatte in Deutschland (und anderen europäischen Ländern) verstärkt auf die größte religiöse Minderheit: die Muslime. Die Forderung nach kultureller Integration gewinnt zunehmend an Bedeutung, wird doch die kulturelle Diversität als Bedrohung für eine definierbare, klare nationale Identität gesehen. Dies liegt vor allem daran, dass nationale Identität in Deutschland nicht heterogen gedacht wird - es fehlt dafür ein politisches Leitbild.

Besonders die kritische Migrationsforschung, aber auch Migrantenselbstorganisationen und involvierte Einzelpersonen distanzierten sich in den letzten Jahren zunehmend vom Integrationsbegriff. Mit der Kritik geht auch der Wunsch einher, den im Jahr 2005 etablierten Begriff »Menschen mit Migrationshintergrund« wieder abzuschaffen, wie dies etwa Klaus Bade gefordert hat. Dabei handelt es sich keineswegs um kleinliche Begriffsdebatten. Vielmehr stehen diese Diskussionen exemplarisch für viel breitere gesellschaftliche Aushandlungsprozesse um Zugehörigkeit, Wandel der nationalen Identität und die Kernfragen heterogener postmigrantischer Gesellschaften: Wie wollen wir miteinander leben, wer gehört zum nationalen Kollektiv und was hält immer diverser werdende Gesellschaften im Kern zusammen? Da der Integrationsbegriff immer noch nur selten gesamtgesellschaftlich gedacht und auf die Perspektive der Bringschuld seitens der Zugewanderten und ihrer Nachkommen verengt wird, wurde in den letzten Jahren wiederholt gefordert, den Begriff zu ersetzen, z.B. durch den Begriff der Inklusion. Weil jedoch der Inklusionsbegriff im öffentlichen Verständnis mit Menschen mit Behinderung verbunden wird, gelingt die Ausweitung des Inklusionsbegriffes derzeit noch nicht. Außerdem bleibt die Frage offen, ob eine Abschaffung oder Ächtung des Integrationsbegriffes letztlich sinnvoll ist, wenn die Strukturen, die ihm zugrunde liegen, erhalten bleiben. Böcker, Goel und Heft haben dies in ihrer kritischen Reflexion zum Integrationsbegriff bereits verworfen: »Der Gewalttätigkeit des Integrations-Diskurses kann nicht durch die Wahl eines alternativen Begriffes entgegengewirkt werden. Weniger das Wort Integration ist problematisch, sondern die dem Diskurs zugrundeliegenden rassistischen Ausgrenzungen, die mit jeder unkritischen Rede von Integration reproduziert werden« (Böcker/ Goel/Heft 2010). Ziel muss daher die Verschiebung des Blickes sein: Weg von den Defiziten, die den Minderheiten zugeschrieben werden, hin zu jenen, die Teilhabestrukturen dadurch erschweren, dass sie die Strukturen dafür nicht schaffen oder blockieren. Ziel ist daher eine Ausweitung des Integrationsbe- 
griffs auf die gesamte Gesellschaft und eine kritische Reflexion des Begriffes, dem stets ein politisch technokratischer Anspruch anhaftet und dem die emotional-affektive Komponente fehlt. Diese wiederum ist im Begriff des Konvivialismus enthalten. Warum also nicht eine Kopplung zwischen moral-philosophischer und politisch-funktionalistischer Konzepte hin zu einer »konvivialen Integration «? Integrationskonzepte, die sich regulierend auf heterogene Gesellschaften beziehen, könnten mit erweiterten, konvivialen Assoziationen verknüpft werden: Es könnte versucht werden, den Begriff der Integration im konvivialistischen Sinne zu öffnen, um die Essentialisierung kultureller Identitätskonstruktionen, die derzeit mit dem Integrationsbegriff einhergeht, aufzubrechen Abgrenzungen von Gruppen zueinander beweglich zu machen, zu verschieben und aufzulösen. Diese Idee könnte dem in Deutschland negativ etablierten gesellschaftspolitischen, technokratischen und einseitig orientierten Konzept der »Integration « als normative Forderung eine emotional-affektive Ausweitung geben und somit das eingangs erwähnte Paradoxon zwischen kognitiver Erkenntnis und affektiver Verweigerung der Einwanderungsrealität neu angehen. Vor allem könnte die konviviale Integration einen Kompass für eine gesamtgesellschaftliche Politik im postmigrantischen Deutschland bilden, in dem eine Anerkennung der Migrationsrealität stark verspätet erfolgt ist und nun zu einer Neuverhandlung von rechtlichen, gesellschaftlichen und politischen Positionen im Anschluss an erfolgte Migrationen und die Selbstanerkennung Deutschlands als einer Einwanderungsgesellschaft führt.

Auf einer Veranstaltung zum zehnjährigen Jubiläum des deutschen $\mathrm{Zu}$ wanderungsgesetzes im April 2015 sagte die Beauftragte der Bundesregierung für Migration, Flüchtlinge und Integration, Aydan Özoguz, sinngemäß< dass wir eine integrative Politik für 81 Millionen Menschen in Deutschland und nicht mehr für 16 Millionen Menschen mit Migrationshintergrund brauchen. Damit macht sie klar, dass die Frage, wie wir in diesem Land zusammenleben wollen, alle Menschen gleichermaßen betrifft. Hier ist ein Paradigmenwandel zu beobachten, der die statische Verwobenheit des Integrationsbegriffs mit dem Migrantischen auch in der Politik dokumentiert.

Konviviale Integration lässt sich somit neu definieren als ein gesellschaftsstrukturierendes Leitmotiv, das sich aus den Teilsegmenten Anerkennung, Chancengerechtigkeit und Teilhabe zusammensetzt mit dem Ziel, Diskriminierung und gesellschaftliche Ungleichheit zu überwinden. Darüber spannt sich das sinnstiftende Narrativ, dass ein Zusammenspiel dieser moralphilosophischen Prinzipien nicht nur durch Perspektiven und Handlungsstrategien der Akteure »vor Ort« gelingt. Der Konvivialismus konzentriert sich zu wenig auf das politische System als strukturgebende und strukturverändernde Instanz und zu stark auf die Strategien des Zusammenlebens von Akteuren und Gruppen: »Wie leben diese zusammen, wie gelingt ihnen das oder eben auch nicht?«, ist die Kernfrage des Konvivialismus. »Welche Rolle 
kommt bei der Strukturierung des Zusammenlebens dem politischen System zu und welche Strukturveränderung muss von der Politik eingefordert werden, damit der gesellschaftlichen Zusammenhalt gestärkt wird?«, sind aber konkrete Fragen, die von der Integrationspolitik gestellt werden. Insofern wäre eine Verzahnung der beiden Konzepte zielführend. Konvivialität geht eher von einem Prozess aus, in dem sich gleichberechtigte Elemente zusammenfügen, aber auch unterscheiden, überschneiden und Grenzen ziehen. Der Antrieb und die Dynamik erscheinen in der Beschwörung und Benennung von maximaler Vielheit zu liegen. Damit aber die Anerkennung und das Hochhalten maximaler Vielheit nicht die Gefahr in sich tragen, irgendwann auch soziale Ungleichheit als Teil der Vielfaltserzählung zu sehen, muss ein sinnstiftender Endpunkt formuliert werden und ein politischer Weg der Umsetzung gefordert werden.

Die Großerzählung, die hinter der konvivialen Integration stehen könnte, hätte zum Inhalt, wie es gelingen könnte, vor dem Hintergrund wachsender Ungleichheit und einer wachsenden kulturellen Diversität - also einer zunehmenden Super-Heterogenisierung - eine Gesellschaft norm- und sinngebend zusammenzuführen und Zugehörigkeit und Identifikation für alle Bürger zu schaffen, und zwar einschließlich der Neu-Bürger. Die Gesellschaft muss sich als »Einheit der Verschiedenen« erzählen, wie dies auch Bundespräsident Joachim Gauck in seiner Rede zur 65-jährigen Feier des deutschen Grundgesetzes getan hat. Integration in ein vielfältiges Kollektiv oder Integration in ein kollektives Narrativ bedeuten allerdings immer die Einfügung von neuen Elementen in eine vermeintlich schon bestehende Entität. Daher müsste parallel auch diese Entität neu erzählt werden. Wenn die Entität die Nation war, das Volk, die nationale Identität, das deutsche Wir - so muss es Ziel der konvivialen Integration sein, genau diese Entitätsbestandteile im Sinne eines integrativen Zusammenlebens neu zu erzählen: Was bedeutet Nation, Volk, deutsche Identität - jetzt wo immer mehr Menschen für sich in Anspruch nehmen dazuzugehören, auch wenn ihre Vorfahren nicht hier geboren sind und ihre Namen unvertraut klingen? Die Herausforderung liegt darin, dieses narrative Kollektiv in seiner Heterogenität zu einer authentischen Identität zu führen, die auf Vielheit gründet. Konviviale Integration wäre dann das leitende Motiv einer durch Vielfalt gekennzeichneten Gesellschaft, die nicht mehr aus Migranten und immer schon Dagewesenen besteht, sondern postmigrantisch den Blick ausweitet und Ungleichheiten in der Gesellschaft adressiert. Dabei wäre Integration der Weg und Konvivialität der sinnstiftende Endpunkt. Konvivialität würde dabei verstanden als Zusammenleben vieler in einer Einheit, die zunächst noch nationalstaatlich strukturiert ist, aber mit einer inklusiven Erzählung einer Nation, für die Pluralismus und Diversität konstitutiv sind. Dies ist eine politische Herausforderung, die parallel zu strukturellen Integrationsmaßnahmen narrativ flankiert werden muss. 
Das Wort Integration taucht im konvivialistischen Manifest an keiner Stelle auf. Die Autoren schreiben auf S. 63: »Jeder Einzelne darf hoffen, dass ihm eine ebenso große Würde zuerkannt wird wie allen anderen Menschen und dass ihm hinreichende materielle Bedingungen zuganglich sind, um seine Auffassung vom guten Leben, unter Berücksichtigung der Auffassungen anderer, $\mathrm{zu}$ verwirklichen, und sich um die Anerkennung der anderen zu bemühen, indem er, wenn er es wünscht, am politischen Leben und an allen Entscheidungen teilnimmt, die seine Zukunft und die seiner Gemeinschaft betreffen.« Es würde sich lohnen, diese Definition in den neuen, auf die Gesamtgesellschaft ausgeweiteten und als Leitmotiv definierten konvivialen Integrationsbegriff einzubauen.

\section{Anerkennung, Chancengerechtigkeit und Teilhabe (ACT) IN DER KONVIVIaLEN DemoKRatie}

Die Autoren des Manifests beschreiben das »Prinzip der gemeinsamen Sozialität: Die Menschen sind gesellschaftliche Wesen, deren größter Reichtum in ihren sozialen Beziehungen besteht.« (S. 61) In postmigrantischen Gesellschaften werden soziale Beziehungen besonders über Minderheitenrechte und -positionen offensiv ausgehandelt sowie Fragen nach nationaler Identität, Zugehörigkeiten, Privilegien und Repräsentationen neu gestellt. Die Frage nach dem »Wer sind wir? « tritt in diesen Gesellschaften, die von einem Migrationsdiskurs geprägt sind, stärker in den Vordergrund. Dabei geht es auch um die Sicherung von Privilegien, wenn zunehmend Menschen, die andere Hautfarben oder Namen haben, mit beanspruchen, Teil des nationalen Kollektivs zu sein, tritt die Herausforderung der Demokratie, ihr Versprechen der Gleichheit und Gleichwertigkeit zu erfüllen, deutlich zu Tage. Dabei kämpfen nicht nur Migranten und ihre Nachkommen um mehr Anerkennung, Chancengerechtigkeit und Teilhabe, sondern gemeinsam mit ihnen große Teile der nicht-migrantischen Bevölkerung, denn Minderheitenrechte werden als ein Basiselement demokratischer Gesellschaften verstanden.

Die Gesellschaftsbeziehungen zwischen Personen mit und ohne Migrationshintergrund sind zunehmend verwoben. Allein 35 Prozent der deutschen Bevölkerung haben bereits in der Verwandtschaft Menschen mit Migrationshintergrund, wodurch sich migrantische Bezugspunkte in der Gesellschaft ausweiten (Foroutan et al. 2014). Durch familiale Bezugspunkte, durch Freundschaftsbeziehungen, Arbeits- und Nachbarschaftskontakte entstehen Interaktionen, neues Wissen, Empathie, Perspektivwechsel und Positionierungen in der Gesellschaft. Sie entstehen aber nicht nur durch Kontakt sondern auch durch ein neues Narrativ, welches die Einwanderungsgesellschaft als konstituierend sieht und dementsprechend als legitime Basis des Nationalstaates, der 
sich als Einwanderungsgesellschaft neu erzählt, aber die alte Erzählung der demokratischen Verfasstheit hochhält, worin nun die Erzählung der Einheit in Vielfalt mündet. Es bilden sich in dieser Gesellschaft der Vielfalt postmigrantische Allianzen, die über die subjektive Bezugsebene hinausgehen und sich vor allem anhand einer Haltung zur offenen Demokratie - zunehmend symbolisiert durch die Haltung zu Minderheitenrechten - definieren und nicht anhand einer migrantischen oder nicht-migrantischen Herkunft.

Die Politik richtet sich in Deutschland zehn Jahre nach den Kämpfen um das deutsche Zuwanderungsgesetz, welches das Land auch juristisch zu einem Einwanderungsland machte, neu aus und eröffnet Perspektiven jenseits des Migrantischen. Die postmigrantische Perspektive auf das ganze Land wird von der Dynamik getragen, dass Anerkennung, Chancengerechtigkeit und Teilhabe - als Grundsäulen einer konvivialen Integration - Versprechen gegenüber allen Bürgern in einer pluralen Demokratie sind und dementsprechend alle Bürger diesbezüglich ansprechbar gemacht werden müssen. Wenn wir Konvivialismus als gelungene Form des Zusammenlebens in heterogenen Demokratien betrachten, dann ist Integration nicht länger nur eine Forderung, die ausschließlich an Migranten zu adressieren ist. Konviviale Integration wäre eine system- und strukturgebende Aufgabe, die begleitet wird von einem positiv-affektiven Metanarrativ jenseits homogenisierender und hyper-individualistischer Perspektiven. Das Metanarrativ formuliert durch die Ausweitung der Perspektive über das Migrantische hinaus einen durch Integrationspolitik zu strukturierenden Zwischenraum für die gesamte Gesellschaft. In der Auseinandersetzung mit diesem Zwischenraum werden auch die Ränder wieder dialogisch involviert. Es geht in diesem politischen Projekt der konvivialen Integration auf dem Weg zu einer konvivialen Demokratie nicht darum, Menschen in diesem Zwischenraum aufzulösen. Die Frage, die sich dem konvivialen Projekt durchgängig stellt, ist, wie sich in einer individualisierten Welt ein Kollektiv bzw. ein kollektives Band schaffen lässt ohne zugleich die Individualität aufzugeben. Wie ist es möglich, die Verschiedenheit aller einzelnen Identitätskonstruktionen und -aushandlungen anzuerkennen, keine auszuschließen, Ambiguitätstoleranz zu fördern und gleichzeitig den Fokus auf eine politisch heterogene, nicht-ausschließende, kollektive Narration zu richten?

Diese Aufgabe könnte durch das politische Projekt der konvivialen Integration gelöst werden - nämlich mit der Zieldefinition, Anerkennung, Chancengerechtigkeit und Teilhabe (ACT) zu gewährleisten. Dadurch ließe sich ein integrativer Zwischenraum erzeugen, um den sich die Politik, aber auch die gesellschaftliche Aushandlungsdynamik suchend herumbewegt, um in dieser diskursiven Arena langsam und kontinuierlich die Vielfalt als Mitte der Gesellschaft erkennbar zu machen und nicht als konfliktives, migrantisches Randphänomen. So entstünde - in Anlehnung an Hannah Arendt - ein politischer Prozess rund um die Auseinandersetzung mit diesem Zwischenraum, 
der sowohl verbindet als auch trennt aber verhindert, dass eine Integration weiterhin als Assimilation in einen Kern von bereits Etablierten wahrgenommen wird. Die konviviale Integrationspolitik lebt nicht davon, dass von Beginn an Gleiche mit Gleichen zusammenkommen und die Ränder sich noch stärker polarisieren. Sie lebt vielmehr von unterschiedlichen und verschiedenen Positionen von Einzelnen, die mit diesen Differenzen leben. Allein, dass es aber der Verständigung bedarf, bezeugt Differenz unter Menschen. Die konviviale Integration entsteht analog dazu nicht mehr »um mich herum«, auch nicht »um uns herum«, sondern »zwischen uns«. Das »Zwischen« konstruiert hierbei ein ambivalentes Gemeinsames, das die Heterogenität der postmigrantischen Gesellschaft reflektiert und gleichzeitig eine Verbindung schafft, ohne aber die Distanz aufzugeben: Weil es »zwischen « uns steht, sind wir nicht unmittelbar miteinander verbunden; da wir uns aber gemeinsam auf dieses Zwischen, unsere »gemeinsame Welt« beziehen (Jaeggi 1997), stehen wir in einem über Sprache und verschiedene Arten von Äußerungen vermittelten Kontakt zueinander. Diese Vermittlung ist von eminenter Bedeutung: Sie bedeutet die Herstellung von Solidarität und erzeugt politische Strukturvorgaben - in dem Fall, die drei Ziele der konvivialen Integration umzusetzen: Anerkennung, Chancengerechtigkeit und Teilhabe (ACT).

Hier müssen allerdings außerhalb der abstrakten Moralität dieser Worte, die mit Sicherheit von den meisten Menschen geteilt werden, in der Tat konkrete Handlungsziele eingefordert und konkrete Aufgaben für Politik und Zivilgesellschaft formuliert werden, die den Zwischenraum in den kommenden Jahre zu einer umkämpften Zone für ACT machen. Die konviviale Integration muss mit Forderungen antreten, um ACT zu konkretisieren: Es braucht eine Schärfung der Antidiskriminierungspolitik zur Stärkung der Anerkennung, wir brauchen gezielte Umverteilungsmaßnahmen zum Abbau von Ungleichheiten und zur Gewährleistung von Chancengerechtigkeit und wir müssen Quoten fordern, zur Sicherstellung der Teilhabe. Folgen den positiv affektiv besetzen Worten keine konkreten Handlungsforderungen, entsteht das, was wir in den Kämpfen nicht nur mit Rechtspopulisten derzeit beobachten können: Der Vorwurf des Gutmenschentums mit abstrakten Weltfriedensträumen. Wir müssen aber den Kampf in der Arena suchen, wenn wir ein konstruktives Angebot machen wollen.

\section{LITERATUR}

Beigang, Steffen/Canan, Coşkun/Foroutan, Naika/Kalkum, Dorina/Schwarze, Benjamin (2015): Deutschland postmigrantisch II - Einstellungen von Jugendlichen und jungen Erwachsenen zu Gesellschaft, Religion und Identität, Berlin: Humboldt Universität. 
Böcker, Anna/Goel, Urmila/Heft, Kathleen (2010): »Integration«, in: Antje Lann Hornscheidt/Adibeli Nduka-Agwu (Hg.): Rassismus auf gut Deutsch - Ein kritisches Nachschlagewerk zu rassistischen Sprachhandlungen, Frankfurt a.M.: Brandes \& Apsel, S. 304-310.

Brähler, Elmar/Decker, Oliver/Kiess, Johannes (2014): Die stabilisierte Mitte. Rechtsextreme Einstellung in Deutschland 2014, Leipzig: Universität Leipzig.

Jaeggi, Rahel (1997): Welt und Person. Zum anthropologischen Hintergrund der Gesellschaftskritik Hannah Arendts, Berlin: Lukas Verlag.

Kühn, Heinz (1979): Stand und Weiterentwicklung der Integration der ausländischen Arbeitnehmer und ihrer Familienangehörigen in der Bundesrepublik Deutschland. Memorandum des Beauftragten der Bundesregierung, Bonn.

Sachverständigenrat deutscher Stiftungen für Integration und Migration (SVR) 2014: Deutschlands Wandel zum modernen Einwanderungsland. Jahresgutachten 2014 mit Integrationsbarometer, Berlin.

Sutterlüty, Ferdinand (2010): In Sippenhaft. Negative Klassifikationen in ethnischen Konflikten, Frankfurt a.M./New York: Campus.

The German Marshall Fund of the United States (GMF) (2013): Transatlantic Trends - Key Findings 2013, online verfügbar unter: www.ui.se/upl/ files/96123.pdf (letzter Zugriff: 12.06.2015). 